\title{
Considering start-ups and shutdowns using an optimisation tool - Including a dairy production planning case study
}

\author{
Magnus Karlsson and Nawzad Mardan
}

\section{Linköping University Post Print}

\section{Tweet}

N.B.: When citing this work, cite the original article.

Original Publication:

Magnus Karlsson and Nawzad Mardan, Considering start-ups and shutdowns using an optimisation tool - Including a dairy production planning case study, 2013, Applied Energy, (107), 338-349.

http://dx.doi.org/10.1016/j.apenergy.2013.02.050

Copyright: Elsevier

http://www.elsevier.com/

Postprint available at: Linköping University Electronic Press

http://urn.kb.se/resolve?urn=urn:nbn:se:liu:diva-93954 


\title{
Considering start-ups and shutdowns using an optimisation tool - including a dairy production planning case study
}

\author{
Magnus Karlsson*, Nawzad Mardan \\ Department of Management and Engineering, Division of Energy Systems, Linköping \\ University, SE-581 83 Linköping, Sweden
}

\begin{abstract}
There are many different aspects a production-planning model has to be able to handle to make a model adequate for the purpose. One aspect is the handling of start-ups and shutdowns for different processes. The production plan is likely to be changed when considering, for example, a cost connected to the start-up and/or shutdown of processes. Besides costs associated with start-ups and shutdowns, waste may be produced during the start-up and shutdown. However, there is also the possibility of carrying out soft start-ups and shutdowns or limiting the number of start-ups and shutdowns. Thus, start-ups and shutdowns have to be handled in an adequate way in models to produce reliable and accurate results. In optimisation tools, this may be dealt with by introducing certain constraints, including integers. In this paper, the implementation of alternative ways to consider start-ups and shutdowns are presented. This is done in the energy system optimisation tool reMIND, which deals with Mixed Integer Linear Programming (MILP) problems. The purpose of this paper is to show four alternatives to consider start-ups and shutdowns in optimisation models. This involves, in total, almost 50 constraints. Also, a simple dairy case study is included in the paper to visualise the effect of implementing the different alternatives to shutdowns.
\end{abstract}

Keywords: MILP, optimisation, production planning, start-up, shutdown

\section{Introduction}

There are many different aspects to consider in the production-planning process, and there are different types of models that can be used to help with different situations. Simulation tools are widely used in industry as one part in the decision-making process. Decision support also includes risk management, economic calculations and optimisation. The decision support is a part of the decision-making process, which means helping people to make good decisions by understanding and analysing the effects of all of the alternatives [1]. Simulation modelling tools may be complemented by other modelling tools that enhance the support for decisions. Mardan and Klahr [2] have shown that an optimisation tool may be a very powerful

\footnotetext{
* Corresponding author. Tel.: +46-13-285739; fax: +46-13-281788.

E-mail address: magnus.karlsson@liu.se (M. Karlsson), nawzad.mardan@liu.se (N. Mardan).
} 
complement to a simulation tool. By combining these tools this may increase the reliability of the results, but it also enhances the possibility of finding better solutions. An optimisation tool may be used to find the optimal result and a simulation tool may be used to find out whether it is possible to run the solution in reality [3].

To further increase the advantages of using a simulation tool and an optimisation tool in combination, it is necessary to consider start-ups and shutdowns in the optimisation tool. Many of the differences in the results from the two different tools occur because of a lack of modelling start-ups and shutdowns in the optimisation tool. Even without the use of both tools in combination there is a need for optimisation tools to consider start-ups and shutdowns, to make adequate production planning.

Many articles have been published on the optimisation of systems, where the costs associated with the starting up and/or shutting down of processes are considered, see for example [4-8]. However, appropriate or detailed information of the type of constraints that are needed to be included in the model for representing a start-up and/or a shutdown process are missing. Furthermore, the papers consider just a start-up and/or a shutdown that is associated with a cost, and do not take into consideration any other alternatives, such as the production of waste (called "waste" in the subsequent text) when shutting down or starting up a process or a restriction in processes requirements that force the processes to start or stop slowly. In addition, neither production planning nor manufacturing industries are used as case studies in published articles when considering start-ups or shutdowns.

When looking at earlier systems that have been modelled using the energy systems optimization tool reMIND [9], scrutinising other energy systems' optimisation tools and conducting an additional survey between companies, four different alternatives on start-ups and shutdowns have been found to be most relevant involving e.g. costs and wastes associated with start-ups and shutdowns.

The purpose of this paper is to show possible ways to model start-ups and shutdowns in optimisation models. Four alternatives have been included in this paper and the corresponding constraints for each alternative are presented. Also, a simple case study is included in the paper to show how important it is to consider start-ups and shutdowns in models for production planning in a dairy. To reduce the size of the paper, only shutdown examples are included, as both start-ups and shutdowns work in the same way.

\section{Method}

\subsection{MILP in general}

As the basis for the study, MILP is used. A MILP problem is defined, in general, according to equations 1 and 2 [10]. The parameters, variables and subscripts found in the constraints below are explained the first time they appear in the text. A nomenclature is also included as help. 
Objective:

$$
\min \sum_{v=1}^{V} \sum_{w=1}^{W}\left(c_{1, v} x_{v}+c_{2, w} y_{w}\right)
$$

subject to:

$$
\begin{gathered}
\sum_{v=1}^{v} \sum_{w=1}^{W}\left(c_{3, v, z} x_{v}+c_{4, w, z} y_{w}\right)\left\{\begin{array}{l}
\leq \\
\geq \\
\geq
\end{array}\right\} c_{z}, \forall z=1,2, \ldots, Z \\
x \geq 0
\end{gathered}
$$

where $v$ and $w$ are the numbers of a specific real and integer variable, respectively, and $V$ and $W$ are the total numbers of real and integer variables, respectively, in the problem. $c$ is a parameter, while $x$ represent continuous variables and $y$ represent the integer variables. $z$ is the number of a specific constraint while $Z$ is the total number of constraints in the problem

In Equation 1 variables are included that influence the system cost and for each variable the corresponding coefficient are included, representing the price per unit of the specific variable. A variable may e.g. be the amount of electricity that is needed to be purchased in the system that is modelled and the corresponding coefficient represents the electricity price. The variables are also included in the constraints. The constraints (included in the model by Equation 2) may be of any kind that restricts the allowed solution range. A constraint may for example represent a limitation of the maximum electricity that may be purchased.

\section{2 reMIND in general}

This study uses the energy system optimisation tool reMIND, which is based on the MIND method (Method for analysis of INDustrial energy systems) developed at Linköping University in Sweden [9, 11]. The method has been developed for the optimisation of dynamic industrial energy systems, but other types of systems may be analysed as well. The dynamics of the modelled systems are considered by dividing time into time steps (TSs). The amount and length of the TSs depend on the purpose of the analysis and the system to be modelled. The structure of the system is modelled using branches and nodes. The branches represent different kinds of flow (e.g. materials and electricity) and the nodes represent different kinds of processes (e.g. separators and boilers). All limitations and relations in the system are included in a standardised file. The file is optimised using an optimisation solver. In this case study, the optimisation solver CPLEX [12] is used. This method minimises the system costs, using MILP, where costs for material and energy are included. The reMIND tool is used more frequently for production planning. Originally, reMIND was built to be a tool for more strategic and long-term analysis, involving less detailed models, and the modelling of start-ups and shutdowns were not crucial. 


\subsection{Alternatives that model start-ups and shutdowns}

There are different ways to model start-ups and shutdowns. In this paper, four alternatives are considered.

- Alternative 1: soft start-ups and soft shutdowns.

- Alternative 2: a cost associated with start-ups and shutdowns.

- Alternative 3: limited amounts of start-ups and shutdowns.

- Alternative 4: a waste associated with start-ups and shutdowns.

For each alternative, both the start-up and shutdown alternative may be used at the same time. The different alternatives may also be included in the same model. The constraints constructed for each alternative are shown in the appendix. The Graphical User Interface (GUI), to include the input data in reMND for the different alternatives, is shown in Figure 1.

\section{Case study model description}

The energy system optimisation tool reMIND is used to model the system. The modelled system in the case study represents a dairy and it is represented as a network of nodes and branches with different colours (the square boxes and the arrows, respectively, found in Figure 2). The nodes represent different processes, buffers, tanks etc., while the branches represent a flow of any kind, such as milk, steam and electricity.

In this model, the yellow branches represent electricity flow, the red branches represent steam flow, the white branches represent a flow of milk products and the blue branches represent a flow of ice water. Ice water is used to cool the milk and is produced using electricity in a cooling machine. Electricity is also used for homogenising, separating skimmed milk from cream, as well as running the packaging machines. The electricity prices fluctuate during the day: higher prices during the day and lower prices during the evening and at night (see figures 3-6). The prices are made up, but are based on daily trends and changes in real spot prices. Steam is used for heating milk products in the pasteurisers. Input data for processes included in the model is presented in Table 1 . For more detailed information regarding input data and the model, please see $[9,13]$.

The production process begins when the whole milk enters the top of the model and goes through different processes and tanks before entering the packaging machines at the bottom of the model. A one-day production, divided into 24 time steps, each representing one hour, is included in the model. In total, $48.14 \mathrm{~m}^{3}$ of yoghurt and $39.76 \mathrm{~m}^{3}$ of sour cream are produced over the course of one day.

The objective of the model is to minimise the system costs. The variables that are included in the system cost are the costs of energy and milk. There is no revenue for selling yoghurt and sour cream and the models include only production processes, not support processes. In other words, the electricity used for lighting, ventilation and other support systems are not modelled 
in this system. Due to the fact that there is no change between the models regarding the support processes and production volume (the same amount of products are produced in all models), it is possible to exclude this in the models as the analyses in this paper compares the results from different models.

\section{Analysed alternatives in the dairy}

In order to illustrate how the method is used and investigate the usefulness of using reMIND as a decision support for production planning, and especially to visualise the effect of implementing the shutdowns, different cases were devised. In this paper, the packaging machines are subject to the shutdown alternatives. The different cases are described further in Table 2 (called "alternatives" in the subsequent text). To simplify the case study, only shutdowns have been modelled (both the start-ups and shutdowns works in the same way). The majority of the model built in reMIND, representing the dairy, is based on material and input data from an existing dairy in Sweden [9, 13]. The values used regarding the shutdown function, e.g. the costs for shutdowns and amount of shutdowns, are created to show the use of the function. The existing dairy operates the packaging machines to reduce the losses of milk as much as possible. Alternative 4 represents this situation and three different amounts of wastes have been included in the case study to visualize how the size of the waste influences the system, as in reality the waste differs between different batches. The values for the other alternatives (found below) have been developed to be in the same order as for Alternative 4 . Comparisons between the different alternatives are therefore not central in the case study. Instead the changes in the system depending on whether the shutdown function is included or not, and the influence on the system dependent on the size of the values in the shutdown function, are in focus. Thus general differences on how the systems react within different alternatives are emphasized.

Alternative 1 represents the soft shutdowns of processes. In other words, this alternative shows the processes that are not possible to shutdown immediately, i.e. it may take multiple time steps to carry out the shutdown. For example, if a process is running in a certain time step, the process must run at a certain percentage of the previous flow in the next time step. In this case study, Alternatives $1 \mathrm{~A}, 1 \mathrm{~B}$ and $1 \mathrm{C}$ represent $50 \%, 60 \%$ and $70 \%$ of the flow in the previous time step, respectively. The threshold value is $1 \mathrm{~m}^{3}-$ in other words, the flow cannot be less than $1 \mathrm{~m}^{3}$.

Alternative 2 represents a shutdown of a process that is associated with a cost, where Alternatives 2A, 2B and 2C represent costs of 200, 400 and 600 SEK (9 SEK equals around $1 €)$ respectively for each shutdown. Alternative 3 represents a limitation of shutdowns, where Alternatives 3A, 3B and 3C represent one shutdown, two shutdowns and three shutdowns respectively. Alternative 4 represents a shutdown that is associated with a waste of flow, where Alternatives 4A, 4B and 4C represent a waste of 40, 80 and 120 litres of yoghurt and sour cream respectively for each shutdown. The minimum flow for Alternatives 2 to 4 is $1 \mathrm{~m}^{3}$. 


\section{Results and analysis}

For all the analysed alternatives, there is no production of yoghurt in the packaging machines during the first 5 hours and no production of sour cream in the packaging machines during the first 8 hours. This is due to the fact that the incubation times for these products are 5 hours and 8 hours respectively (see figures 3-6).

The largest electricity consuming procedures, namely the homogenising process, separating process and ice water producing process takes place when electricity prices are low. These electricity consuming units account for $90 \%$ of the total electricity consumption, and the rest is the electricity that is used in the packaging processes. Therefore, to reduce the system cost, the units that consume the most electricity, i.e. the separator, homogeniser and ice water production units, operate when the prices are low. There are indications that the packaging processes run when the electricity prices are low or when the cost of electricity has slightly decreased, even though the amount of electricity used is relatively small.

The detailed overview of the optimisation results for Alternative 1, which represents the soft shutdowns, is shown in Figure 3. As can be seen, the number of shutdowns for the sour cream and yoghurt production processes for the analysed alternatives, Alternatives $1 \mathrm{~A}, 1 \mathrm{~B}$ and $1 \mathrm{C}$, are lower than the Reference alternative. The total number of shutdowns for the Reference alternative and Alternatives $1 \mathrm{~A}, 1 \mathrm{~B}$ and $1 \mathrm{C}$ are 10, 8, 9 and 5 respectively. This is due to the fact that the packaging process needs time to stop as well as to run the process when electricity prices are low. It can be seen that in Alternative 1C, both packaging machines run continuously without shutting down to package the sour cream and yoghurt products. It can also be seen that the packaging machines in this alternative are not running at the maximum capacity, but they are running at almost $70 \%$ of their capacity.

The system costs and the peak demand for steam and electricity are shown in Table 3. The system costs are calculated for one production day. The total steam and electricity demand is the same for all Alternatives (except Alternatives 4A, 4B and 4C, where the shutdowns of the processes are associated with a waste).

Table 3 indicates that the system costs and the electrical and steam peak demand for Alternatives $1 \mathrm{~A}, 1 \mathrm{~B}$ and $1 \mathrm{C}$ increase as a consequence of the altered production-planning. However, the system costs for Alternatives $1 \mathrm{~A}, 1 \mathrm{~B}$ and $1 \mathrm{C}$ have increased only slightly (less than $0.1 \%$ ). The reason why the system cost increases compared to the Reference alternative is due to that a constraint is included in the system. In the Reference alternative it is possible to shut down the packaging machines without considering any type of restriction. However, in reality this is not possible as a shutdown of the packaging machines involves some type of impact, such as e.g. producing a waste or a generating a cost.

The results from the optimisation for Alternative 2, which represents shutdowns associated with a cost, can be seen in Figure 4. The results from Figure 4 show that the number of shutdowns in the packaging processes for the analysed Alternatives $2 \mathrm{~A}, 2 \mathrm{~B}$ and $2 \mathrm{C}$ are lower than the Reference alternative. The total number of shutdowns for the Reference alternative is 10 shutdowns and for Alternatives 2A, 2B and 2C there are four shutdowns. Since each 
shutdown of a process is associated with a cost for each process, i.e. the packaging machines are shutdown only once to keep the system costs as low as possible. Whenever possible, the packaging machines operate at times when the electricity price is low. It can also be seen that the maximum level for packaging machines for Alternatives $2 \mathrm{~A}, 2 \mathrm{~B}$ and $2 \mathrm{C}$ is the same as the Reference alternative. In other words, the packaging machine runs at its maximum capacity during certain hours. The system costs and peak demand for electricity and steam are shown in Table 4.

The results from Table 4 show that the system costs for the Alternatives 2A, 2B and 2C increase due to the fact that each shutdown is associated with a cost, and as the costs increase the system costs also increase. As a consequence of the altered production planning, the electrical and steam peak demand for all alternatives are decreased except for Alternative 2B, in the case of steam demand.

The detailed overview of results from the optimisation for Alternative 3, which represents the limitation of shutdown of processes, is shown in Figure 5. This shows that the number of shutdowns for the packaging machines (sour cream and yoghurt production) depend on the limitation. For example, in Alternative 3A there is only one shutdown for the sour cream and yoghurt production processes in each packaging machine, while in Alternatives $3 \mathrm{~B}$ and $3 \mathrm{C}$ there are two and three shutdowns respectively. Figure 5 shows that the maximum level for packaging processes for the Alternatives $3 \mathrm{~A}, 3 \mathrm{~B}$ and $3 \mathrm{C}$ are the same as the Reference alternative. The optimal production procedure takes place when the cost of electricity has slightly decreased. The results from Table 5 show that the system costs for the Alternatives 3A, 3B and $3 \mathrm{C}$ have decreased slightly and depend on the number of shutdowns. It can also be seen that the system cost for Alternative $3 \mathrm{C}$ is almost the same as the Reference alternative. The reason is that the number of shutdowns is approximately the same as the Reference alternative. The electrical and steam peak demand for the Alternatives $3 \mathrm{~A}, 3 \mathrm{~B}$ and 3C fluctuate, sometimes it is smaller than the Reference alternative and other times it is larger, depending on production planning, i.e. when the various processes, such homogenisation and pasteurisation, are running.

The results from the optimisation for Alternative 4, which represent shutdowns that are associated with a waste of yoghurt or sour cream, can be seen in Figure 6. It can be seen that the number of shutdowns in the packaging machines for all alternatives are lower than the Reference alternative. The total number of shutdowns for the Reference alternative is 10 , while Alternatives 4A, 4B and 4C have four shutdowns each. Since each shutdown of a process is associated with a waste, each process, i.e. packaging process, shuts down only once, to keep the system cost as low as possible. The maximum levels for packaging processes for all of the alternatives remain the same as the Reference alternative. In order to decrease the cost of running the packaging machines, the machines operate when the cost of electricity has slightly decreased. The system costs and peak demand for electricity and steam are shown in Table 6.

The results from Table 6 show that the system costs for Alternatives 4A, 4B and 4C increase due to the fact that each shutdown is associated with a waste. The system costs increase when 
the amount of the waste increases. As a consequence of the altered production planning, the electrical and steam peak demand for all alternatives are decreased except for Alternative 4B, in the case of steam demand.

\section{Concluding discussion}

By integrating the possibility of modelling start-ups and shutdowns in an optimisation tool, the results are more reliable and production planning is more likely to be more accurate. The complement of a simulation tool to an optimisation tool will be less important in this regards. However, other issues may motivate a use of the tools in combination, e.g. modelling of production interruptions and idling of production units.

One drawback of including the functionality for start-ups and shutdowns is that large numbers of constraints are needed to illustrate the start-ups and shutdowns correctly. Integers are also needed for the functionality, which might lead to longer solution times. In this simple case study, additionally between 100 and 300 integers has been included, with the lowest value corresponding to Alternative 1 and the highest corresponding to Alternative 4. The problems have a solution time of less than 1 second for almost all models. Only Alternative 1 has longer solution times, up to 17 seconds. However, as Alternative 1 has less integers than the other alternatives, the amount of integers is not the issue which is increasing the solution times, instead, it is the complexity of the possible solutions for the model that are influencing the length of the solution times. In general, the solution times for the case study can be regarded as moderate.

The case study has shown robust solutions, as the system costs when including the shutdown alternatives, do not increase by more than $0.6 \%$ in the worst-case scenario. Regarding Alternatives 1 and 3 (soft shutdowns and limited amounts of shutdowns), it is shown that it is possible to run the process continuously with only a minor increase in system costs (less than 0.014\%). Alternative 4 (a waste associated with shutdowns) gives a higher cost compared with the other alternatives, as the waste also results in costs being produced in other parts of the process. Alternatives 2 and 4 (a cost associated with shutdowns and a waste associated with shutdowns) show the same tendency of trying to reduce the amount of shutdowns as much as possible.

The peaks for steam and electricity are shown for each alternative. In some of the solutions the peaks increase considerably, and some alternatives increase by almost $60 \%$. As no cost is associated with the peaks there is no incitement to keep them low. However, this has to be considered in future analyses, but as the focus of this paper has been start-ups and shutdowns of processes, this has not been considered here.

\section{Nomenclature}

c a parameter: represents e.g. (1) a slope of a function [slope] and (2) a step in a function [step] (real) 
$e \quad$ the proportion of the flow in the branches, in the previous and/or the following time step, that have to pass the branches in the actual time step (real)

$g \quad$ continuous variable (real, help variable)

$i \quad$ flow entering a node (integer)

I total amount of flows entering a node (integer)

$j \quad$ flow leaving a node (integer)

$J \quad$ total amount of flows leaving a node (integer)

$k \quad$ indicating a binary variable associated with flow(s) (integer)

lts last time step (integer)

$\min \quad$ lower limit value (real)

$o \quad$ objective function

pr price (real)

$r, r 1, r 2 \quad$ indicating a help binary variable (integer)

$R \quad$ total amount of start-ups (R1) or shutdowns (R2) (integer)

$s \quad$ constant cost when the process is in operation (real)

$S \quad$ cost of starting up or shutting down (real)

$t \quad$ time step (integer)

$T \quad$ total amount of time steps (integer)

tv threshold value (real)

$u \quad$ a large number (real) (determined in reMIND, set to $10^{6}$ by default)

$v \quad$ the number of a specific real variable (integer)

$V \quad$ the total number of real variables in the problem (integer)

$w \quad$ the number of a specific integer variable (integer)

W the total number of integer variables (integer)

$w f \quad$ indicating the waste flow (real)

WF a fixed (WF1) or percentage (WF2) value indicating the amount of waste (real) 


$\begin{array}{ll}x & \text { continuous variable, represents a flow of any kind (real) } \\ y & \text { integer variable (integer) } \\ Y & \text { binary variable (only attaining the values } 0 \text { or 1) (integer) } \\ Z & \text { the number of a specific constraint (integer) } \\ Z & \text { total number of constraints (integer) }\end{array}$

\section{Acknowledgements}

We would like to thank the Swedish Energy Agency (SEA) for their financial support. We would also like to thank Mats Söderström, at Linköping University, for his valuable comments on this paper.

\section{Appendix}

"The process", as referred to in the presentation below, is an assumed machine, process or production line that is possible to start-up or shutdown according to the alternatives. The alternatives are constructed so that a start-up or a shutdown is associated with a specific TS. This TS is associated with, for example, a cost or a waste according to the alternative that is included in the process.

There are two constraints that are included in Alternatives 2 to 4 that are exactly the same, namely Constraints 3 and 4 . These constraints are included if both a start-up or a shutdown is included for each alternative. The reason is to make sure that the flows are zero in the TSs when the process is not in operation. Constraint 3 also delimits the possible flows with an upper limit $\left(\mathrm{C}_{\mathrm{u}}\right)$, while Constraint 4 includes a lower limit $\left(\mathrm{C}_{\min }\right)$. The lower limit states the minimum flow through the process, which must be larger than zero.

$$
\begin{aligned}
& \sum_{i}^{I} x_{t, i}-C_{u} \cdot Y_{k, t} \leq 0, \forall t \\
& \sum_{i}^{I} x_{t, i}-C_{\min } \cdot Y_{k, t} \geq 0, \forall t
\end{aligned}
$$

$i$ is the specific number of a flow entering a node and $I$ is the total number of flows. $t$ represent the specific time step for the constraint while $Y_{k, t}$ represents a binary variable for the specific time step $t$. The subscript $k$ indicates a binary variable that is associated with flows and is included to separate the binary variable $Y_{k, t}$ from other binary variables included as help variables in the coming constraints. The binary help variables are indicated by the subscripts $r, r 1$ and $r 2$. 
For Alternatives 2 to 4, it is also possible to include a cost if there is a flow through the process according to Constraint 5.

$$
C_{p r} \cdot \sum_{t=1}^{T} Y_{k, t}=s, s \in O
$$

where $C_{p r}$ indicates the price for running the process and $s$ is the cost when the process is in operation.

Three constraints are repeated in the list of constraints below, to make it easier for the reader to follow the idea of the different alternatives.

\subsubsection{Alternative 1 (Soft start-ups and shutdowns)}

This alternative is included to illustrate processes that are not possible to start-up or shutdown immediately. This means that the processes needs to start-up or shutdown slowly, i.e. increasing (or decreasing) the amount of produced quantities in the process at a certain pace until full production (or no production) may be achieved.

\section{Constraints for the soft start-up alternative:}

Constraint 6 is included to set the proportion of the flow $\left(C_{e}\right)$ that has to pass a process in the preceding TS.

$$
\sum_{i=1}^{I}\left(x_{t-1, i}-C_{e} \cdot x_{t, i}\right)+C_{u} \cdot Y_{r, t} \geq 0, \forall t \geq 2
$$

If the production process exceeds the threshold value $\left(C_{t v}\right)$, the process is running in the preceding TS, otherwise it is closed down. Constraints 7 to 10 are included to illustrate this.

$$
\begin{gathered}
\sum_{i=1}^{I} x_{t, i}-g_{1, t}-g_{2, t}=0, \forall t \geq 2 \\
C_{t v} \cdot\left(1-Y_{r, t}\right)-g_{1, t} \leq 0, \forall t \geq 2 \\
C_{u} \cdot\left(1-Y_{r, t}\right)-g_{1, t} \geq 0, \forall t \geq 2 \\
g_{2, t}-C_{t v} \cdot Y_{r, t} \leq 0, \forall t \geq 2
\end{gathered}
$$

To separate continuous variables representing a real flow of any kind $(x)$ from continuous variables used as helping variables in the constraints, the latter are denominated $g$.

If the first start-up is supposed to be accomplished within the analysis period (specified in the GUI), Constraint 11 is included: 


$$
\sum_{i}^{I} x_{t, i}-C_{t v} \leq 0, t=1
$$

\section{Constraints for the soft shutdown alternative:}

Constraint 12 is included to set the proportion of the flow $\left(C_{e}\right)$ that has to pass a process in the subsequent TS.

$$
\sum_{i=1}^{I}\left(x_{t, i}-C_{e} \cdot x_{t-1, i}\right)+C_{u} \cdot Y_{t-1} \geq 0, \forall t \geq 2
$$

If the production in the process exceeds the threshold value $\left(C_{t v}\right)$, the process is running in the subsequent TS, otherwise it is closed down. Constraints 13 to 16 are included to illustrate this.

$$
\begin{aligned}
& \sum_{i=1}^{I} x_{t, i}-g_{1, t}-g_{2, t}=0, \forall t \neg t_{l t s} \\
& C_{t v} \cdot\left(1-Y_{t}\right)-g_{1, t} \leq 0, \forall t \neg t_{l t s} \\
& C_{u} \cdot\left(1-Y_{t}\right)-g_{1, t} \geq 0, \forall t \neg t_{l t s} \\
& g_{2, t}-C_{t v} \cdot Y_{t} \leq 0, \forall t \neg t_{l t s}
\end{aligned}
$$

where the subscript lts is an abbreviation for “Last Time Step”. In Constraints 13 to 16 constraints for the last time step is thus not created.

If the last shutdown is supposed to be accomplished within the analysis period (specified in the GUI), Constraint 17 is included:

$$
\sum_{i}^{I} x_{t, i}-C_{t v} \leq 0, t=l t s
$$

\subsubsection{Alternative 2 (A cost associated with start-ups and shutdowns)}

To illustrate the cases where there is a cost associated with start-ups and shutdowns, the following alternative should be used.

\section{Constraints for the start-up cost alternative:}

Constraints 18 to 21, as well as 3, 4 and 5, are needed.

Constraint 18 is included to make sure that if the process is in operation in TS, then the binary help variable $Y_{t, r}$, is also activated.

$$
Y_{k, t}-Y_{r, t} \leq 0, \forall t
$$


Constraint 19 is included to determine a cost $\left(S_{t}\right)$ for the start of the process, where the price for the start of the process is set by $C_{p r} . Y_{t-1, k}$ indicates whether the process is in operation in the previous time step.

$$
C_{p r} \cdot\left(Y_{r, t}-Y_{k, t-1}\right)-S_{t}=0, \forall t
$$

Constraint 20 is included to make sure that the difference within the parenthesis in Constraint 19 remains larger than or equal to zero.

$$
Y_{k, t-1}-Y_{r, t} \leq 0, \forall t
$$

The total costs of start-ups for all time steps $(S)$ are inserted in the objective function $(o)$ according to Constraint 21.

$$
\sum_{t=1}^{T} S_{t}=S, S \in O
$$

where $T$ is the total amount of time steps.

If a start cost is to be included for the first time step this is stated in the GUI and the $Y_{k t-1}$ is set to zero.

\section{Constraints for the shutdown cost alternative:}

Constraints 22 to 25, as well as 3, 4 and 5, are needed.

Constraint 22 is included to make sure that if the process is in operation in TS, then the binary help variable $Y_{r, t}$ is also activated (Constraint 22 is the same as Constraint 18).

$$
Y_{k, t}-Y_{r, t} \leq 0, \forall t
$$

Constraint 23 is included to determine a cost $\left(S_{t}\right)$ for a shutdown of the process, where the price for the shutdown of the process is set by $C_{p r}$. $Y_{t+1, k}$ indicates whether the process is in operation in the subsequent time step.

$$
C_{p r} \cdot\left(Y_{r, t}-Y_{k, t+1}\right)-S_{t}=0, \forall t
$$

Constraint 24 is included to make sure that the difference within the parenthesis in Constraint 23 remains larger than or equal to zero.

$$
Y_{k, t+1}-Y_{r, t} \leq 0, \forall t
$$

The total costs of the shutdowns for all of the time steps are inserted in the objective function according to Constraint 25 (Constraint 25 is the same as Constraint 21).

$$
\sum_{t=1}^{T} S_{t}=S, S \in O
$$


If a shutdown cost is to be included for the last time step, this is stated in the GUI and the $Y_{k, t+1}$ is set to zero.

\subsubsection{Alternative 3 (Limited amounts of start-ups and shutdowns)}

In this alternative, the number of start-ups and shutdowns are limited.

\section{Constraints for the start-up limitation alternative:}

Constraints 26 to 29, as well as 3, 4 and 5, are needed.

Constraint 26 is included to set the amount of start-ups $\left(C_{R 1}\right)$ to be included for the process.

$$
C_{R 1}-\sum_{t=1}^{T} Y_{r, t}\left\{\begin{array}{l}
\leq \\
= \\
\geq
\end{array}\right\} 0
$$

Constraint 27 is included to make sure that the process is not in operation in TS if there is a start-up.

$$
Y_{k, t}+Y_{r, t} \leq 1, \forall t
$$

Constraint 28 is included to make sure that the process is in operation in the subsequent time step of TS, if there is a start-up.

$$
Y_{r, t}-Y_{k, t+1} \leq 0, \forall t
$$

Constraint 29 is included to make sure that the process is in operation in the subsequent time step of TS, making sure that there is a start-up in TS or a flow in TS.

$$
Y_{k, t+1}-Y_{k, t}-Y_{r, t} \leq 0, \forall t
$$

If a start-up in the first time step is to be included in the amount of specified start-ups (specified in the GUI), $t$ starts from zero in equations 26, 28 and 29.

\section{Constraints for the shutdown limitation alternative:}

Constraints 30 to 34, as well as 3, 4 and 5, are needed.

Constraint 30 is included to set the amount of shutdowns $\left(C_{R 2}\right)$ to be included for the process.

$$
C_{R 2}-\sum_{t=2}^{T} Y_{r, t}\left\{\begin{array}{l}
\leq \\
= \\
\geq
\end{array}\right\} 0
$$


Constraint 31 is included to make sure that the process is not in operation in TS if there is a shutdown (Constraint 31 is the same as Constraint 27).

$$
Y_{k, t}+Y_{r, t} \leq 1, \forall t
$$

Constraint 32 is included to make sure that the process is in operation in the preceding time step of TS, if there is a shutdown.

$$
Y_{r, t+1}-Y_{k, t} \leq 0, \forall t
$$

Constraint 33 is included to make sure that the process is in operation in the preceding time step of TS, making sure that there is a shutdown in TS or there is a flow in TS.

$$
Y_{k, t}-Y_{k, t+1}-Y_{r, t+1} \leq 0, \forall t
$$

If a shutdown in the last time step is to be included in the amount of specified shutdowns (specified in the GUI), Constraint 34 is included and $T$ in Constraint 30 is changed to $T+1$ :

$$
Y_{k, t}-Y_{r, t+1} \leq 0, \forall t=l t s
$$

If a shutdown in the first time step is to be included in the amount of specified shutdowns (specified in the GUI), i.e. if there is no flow in Time step 1 it is assumed that it is a shutdown, $t=2$ is changed to $t=1$ in equation 30 .

\subsubsection{Alternative 4 (A waste associated with start-ups and shutdowns)}

Each start-up or shutdown is associated with a waste flow in this alternative. The waste may be calculated in two different ways: (1) "Fixed waste" means that the amount wasted does not depend on the production volume and (2) "Percentage waste" means that the amount wasted is dependent on the production volume. The waste created $\left(x_{w f, t}\right)$ is delivered to a specific branch (flow) in the model.

\section{Constraints for the start waste alternative:}

Constraints 35 to 37, as well as 3, 4 and 5, are needed. Also, one or more constraints from Constraints 38 to 40 are needed.

Constraint 35 is included to indicate that the process is in operation (using $Y_{t-1, k}$ ) and to set the binary help variable $Y_{r 1, t-1}$, which is used in the subsequent two constraints.

$$
Y_{k, t-1}-Y_{r 1, t-1} \leq 0, \forall t
$$

Constraint 36 is included to make sure that the process is not in operation in the preceding time step of TS, in joint cooperation with Constraint 37. Constraint 37 is also included to set the binary help variable $Y_{r 2, t-1}$, which, in turn, is used for setting the waste flow in Constraints 38 to 40 . 


$$
\begin{aligned}
& Y_{k, t}-Y_{r 1, t-1} \leq 0, \forall t \\
& Y_{r 1, t-1}-Y_{k, t-1}-Y_{r 2, t-1}=0, \forall t
\end{aligned}
$$

Fixed waste or percentage waste alternative (exclusive alternatives):

Fixed waste:

Constraint 38 is included to set a fixed waste flow for flow $\mathrm{x}_{\mathrm{wf}, \mathrm{t}}$. The waste amounts to $\mathrm{C}_{\mathrm{WF} 1}$.

$$
x_{w f, t}-C_{W F 1} \cdot Y_{r 2, t-1}=0, \forall t
$$

Percentage waste:

The constraints are divided into two different exclusive alternatives representing a batch process and a continuous process respectively:

Alternative A (batch process):

Constraint 39 is included to set a percentage waste flow for flow $x_{w f, t} . C_{W F 2}$ represents a percentage value.

$$
x_{w f, t}-C_{W F 2} \cdot \sum_{i}^{I} x_{t, i}+C_{u} \cdot\left(1-Y_{r 2, t-1}\right) \geq 0, \forall t
$$

$x_{t, i}$ represents flows entering the node with the specific resource.

Alternative B (continuous process):

Constraint 40 is included to set a percentage waste flow for flow $x_{w f, t}$. $C_{W F 2}$ represents a percentage value.

$$
x_{w f, t}-C_{W F 2} /\left(1-C_{W F 2}\right) \cdot \sum_{j}^{J} x_{t, j}+C_{u} \cdot\left(1-Y_{r 2, t-1}\right) \geq 0, \forall t
$$

$x_{t, j}$ represents flows leaving the node with the specific resource. $j$ indicates a flow leaving the node and $J$ is the total amount of flows leaving the node.

The waste flow $\left(x_{w f, t}\right)$ is a flow leaving the node but is not a flow of the kind denominated $x_{t, j}$.

\section{Constraints for the shutdown waste alternative:}

Constraints 41 to 43, as well as 3, 4 and 5, are needed. Also, one or more constraints from Constraints 44 to 46 are needed.

Constraint 41 is included to indicate that the process is in operation (using $Y_{t, k}$ ) and to set the binary help variable $Y_{r 1, t}$, which is used in the subsequent two constraints.

$$
Y_{k, t}-Y_{r 1, t} \leq 0, \forall t
$$


Constraint 42 is included to make sure that the process is not in operation in the subsequent time step of TS, in joint cooperation with Constraint 43. Constraint 43 is also included to set the binary help variable $Y_{r 2, t}$, which, in turn, is used for setting the waste flow in Constraints 44 to 46.

$$
\begin{aligned}
& Y_{k, t+1}-Y_{r 1, t} \leq 0, \forall t \\
& Y_{r 1, t}-Y_{k, t+1}-Y_{r 2, t}=0, \forall t
\end{aligned}
$$

Fixed waste or percentage waste alternative (exclusive alternatives):

Fixed waste:

Constraint 44 is included to set a fixed waste flow for flow $\mathrm{x}_{\mathrm{wf}, \mathrm{t}}$. The waste amounts to $\mathrm{C}_{\mathrm{WF} 1}$.

$$
X_{w f, t}-C_{W F 1} \cdot Y_{r 2, t}=0, \forall t
$$

Percentage waste:

The constraints are divided into two different exclusive alternatives representing a batch process and a continuous process respectively:

Alternative A (batch process):

Constraint 45 is included to set a percentage waste flow for flow $\mathrm{x}_{\mathrm{wf}, \mathrm{t}} \cdot \mathrm{C}_{\mathrm{WF} 2}$ represents a percentage value.

$$
x_{w f, t}-C_{W F 2} \cdot \sum_{i}^{I} x_{t, i}+C_{u} \cdot\left(1-Y_{r 2, t}\right) \geq 0, \forall t
$$

$x_{t, i}$ represents flows entering the node with the specific resource.

Alternative B (continuous process):

Constraint 46 is included to set a percentage waste flow for flow $x_{w f, t} . C_{W F 2}$ represents a percentage value.

$$
x_{w f, t}-C_{W F 2} /\left(1-C_{W F 2}\right) \cdot \sum_{j}^{J} x_{t, j}+C_{u} \cdot\left(1-Y_{r 2, t}\right) \geq 0, \forall t
$$

$x_{t, j}$ represents flows leaving the node with the specific resource.

The waste flow $\left(x_{w f, t}\right)$ is a flow leaving the node, but is not a flow of the kind denominated $x_{t, j}$. 


\section{References}

[1] Barry, L. Using the Data Warehouse for Decision Support. Website: http://wwwact.ucsd.edu/dw/forum9806/index.htm 1998. Accessed 09 September 2011.

[2] Mardan, N., Klahr, R. Combining optimisation and simulation in an energy systems analysis of a Swedish iron foundry. Energy 2012; 44(1): 410-419..

[3] Mardan, N., Karlsson, M., Klahr R. Industrial decision-making for energy efficiency combining optimization and simulation. In Proceedings of the 24th International Conference on Efficiency, Cost, Optimization, Simulation and Environmental Impact of Energy Systems, Novi Sad, Serbia 2011; 1,442-1,452.

[4] Velasco-Garcia, P., Varbanov, P. S. Utility systems operation: Optimisation-based decision making. Applied Thermal Engineering 2011; 31(16): 3,196-3,205.

[5] Marcovecchio, M. G., Novais, A. Q., Grossmann, I. E. A deterministic optimization approach for the unit commitment problem. Computer Aided Chemical Engineering 2011; 29: 532-536.

[6] Nazari, M. E., Ardehali, M. M., Jafari, S. Pumped-storage unit commitment with considerations for energy demand, economics, and environmental constraints. Energy 2010; 35(10): 4,092-4,101.

[7] Krüger, K., Franke, R., Rode, M. Optimization of boiler start-up using a nonlinear boiler model and hard constraints. Energy 2004; 29(12-15): 2,239-2,251.

[8] Kong, H., Qi, E., Li, H., Li, G., Zhang, X. An MILP model for optimization of byproduct gases in the integrated iron and steel plant, Applied Energy 2010; 87 (7): 2156-2163 (2010).

[9] Karlsson, M.: The MIND method: A decision support for optimization of industrial energy systems - Principles and case studies. Applied Energy 2011; 88(3): 577-589.

[10] Rardin, R. L. Optimization in operations research. Prentice-Hall Inc., Upper Saddle River, New Jersey, USA 1998.

[11] Nilsson, K. Cost-Effective Industrial Energy Systems - Multiperiod Optimization of Operating Strategies and Structural Choices. Linköping Studies in Science and Technology 1993, Dissertation No. 315, Linköping University, Linköping, Sweden.

[12] CPLEX. Using the CPLEX Callable Library. CPLEX Optimization Inc., Incline Village: Nevada, USA 1995.

[13] Karlsson, M., Mardan, N. Timing and sizing of investments in industrial processes - the use of an optimization tool, ECOS2010, 23rd International Conference on Efficiency, Cost, Optimization, Simulation and Environmental Impact of Energy Systems, Lausanne, Switzerland, Book 4, pp. 263-270, 2010. 


\section{Captions}

\section{Figures}

Figure 1. GUI for the start-up and shutdown alternatives.

Figure 2. A schematic outline of the modelled system derived from reMIND. The branches represent different flows of electricity, steam, ice water, milk and finished product. The nodes represent different processes, tanks and buffers.

Figure 3. Results from the optimisation for the Reference alternative and Alternative 1 for the soft shutdowns. Alternatives $1 \mathrm{~A}, 1 \mathrm{~B}$ and $1 \mathrm{C}$ represent $50 \%, 60 \%$ and $70 \%$ of the previous flow respectively if the process is shut down.

Figure 4. Results from the optimisation for the Reference alternative and Alternative 2, where the shutdown was associated with a cost. Alternative 2A, 2B and 2C represent a cost of 200, 400 and 600 SEK for each shutdown respectively.

Figure 5. Results from the optimisation for the Reference alternative and Alternative 3, which represent the limitation of shutdowns, where Alternatives 3A, 3B and 3C represent one shutdown, two shutdowns and three shutdowns respectively.

Figure 6. Results from the optimisation for the Reference alternative and Alternative 4, which represent a shutdown that is associated with a waste flow, where Alternatives 4A, 4B and 4C represent a waste of 40, 80 and 120 litres of yoghurt or sour cream respectively for each shutdown.

\section{Tables}

Table 1. The input data for the different processes in the model [4].

Table 2. The analysed alternatives.

Table 3. System costs and peak demand at the dairy for Alternative 1.

Table 4. System costs and peak demand at the dairy for Alternative 2.

Table 5. System costs and peak demand at the dairy for Alternative 3.

Table 6. System costs and peak demand at the dairy for Alternative 4. 
Start and Stop Equation

Description: Defines a start and stop for the production. Observe that a small difference in the values between the different time steps needs to be included in at least one of the Source functions when using the StartStop Equation. Otherwise there will be an error in the solution.

Label StartStopEquationFu105

$\checkmark$ Alternative 1

V Alternative 2

$\checkmark$ Alternative 3

$\checkmark$ Alternative 4

Type of resource:

Milk

Steam

Ice Water

Flectricitv

\section{Alternative 1 \\ $\nabla$ Start up delay $\square$ Shut down delay \\ Start up within the analysis period \\ Shut down within the analysis period}

\section{Alternative 3}

Start up limitation $\quad \nabla$ Shut down limitation

Proportion of flow: 0.0

Threshold value : 0.0

First time step is included

Last time step is included

Operating cost: 0.0

Minimum flow: 0.0

$\mathrm{R}<\mathrm{R}=\mathrm{R}>\mathrm{Q}<\mathbf{R}<$

0.0

\section{Alternative 2 \\ Start up cost $\quad \nabla$ Shut down cost \\ Start cost for the first timestep}

Start cost : $\quad 0.0$

Stop cost for the last timestep

Stop cost : $\quad 0.0$

Operating cost: 0.0

Minimum flow: 0.0

\section{Alternative 4}

Batch process $\bigcirc$ Continuous process

Start up waste $\quad$ Shut down waste

Start waste for the first timestep

Stop waste for the last timestep

Percentage value

Fixed value :

Operating cost:

0.0

Minimum flow:

0.0

Waste flow:

Figure 1. GUI for the start-up and shutdown alternatives. 


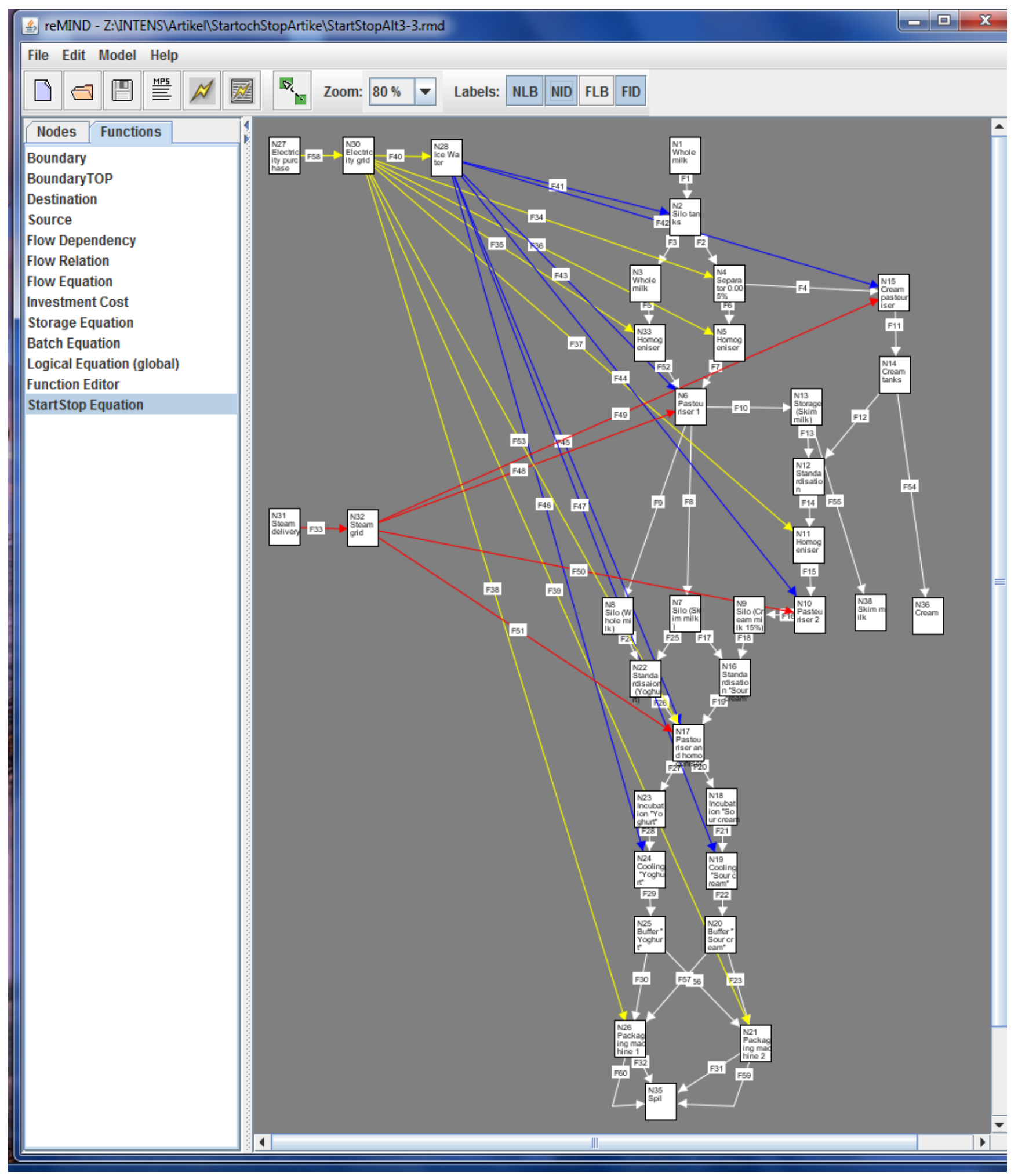

Figure 2. A schematic outline of the modelled system derived from reMIND. The branches represent different flows of electricity, steam, ice water, milk and finished product. The nodes represent different processes, tanks and buffers. 


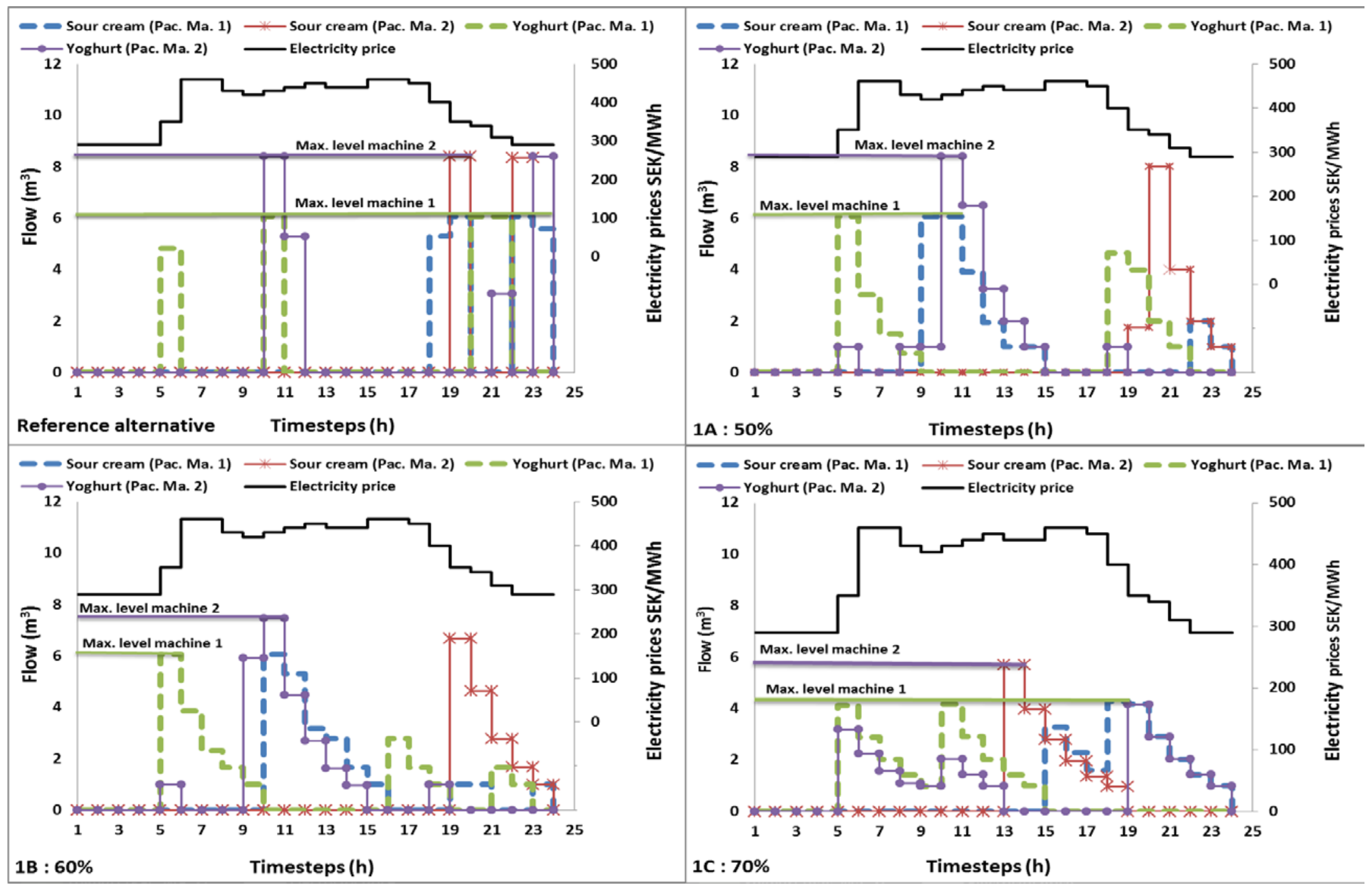

Figure 3. Results from the optimisation for the Reference alternative and Alternative 1 for the soft shutdowns. Alternatives $1 \mathrm{~A}, 1 \mathrm{~B}$ and $1 \mathrm{C}$ represent $50 \%, 60 \%$ and $70 \%$ of the previous flow respectively if the process is shut down. 


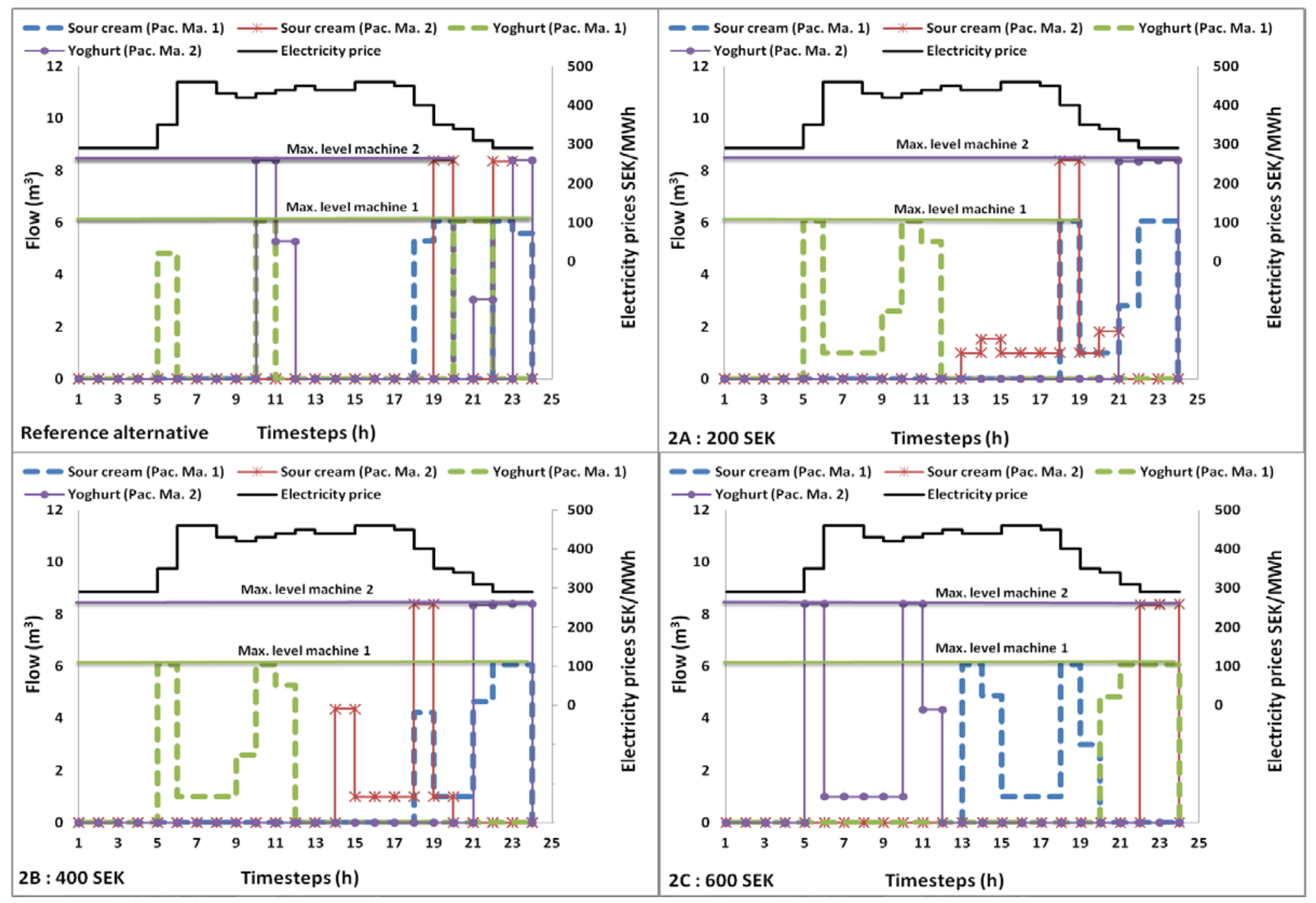

Figure 4. Results from the optimisation for the Reference alternative and Alternative 2, where the shutdown was associated with a cost. Alternative 2A, 2B and 2C represent a cost of 200, 400 and 600 SEK for each shutdown respectively. 


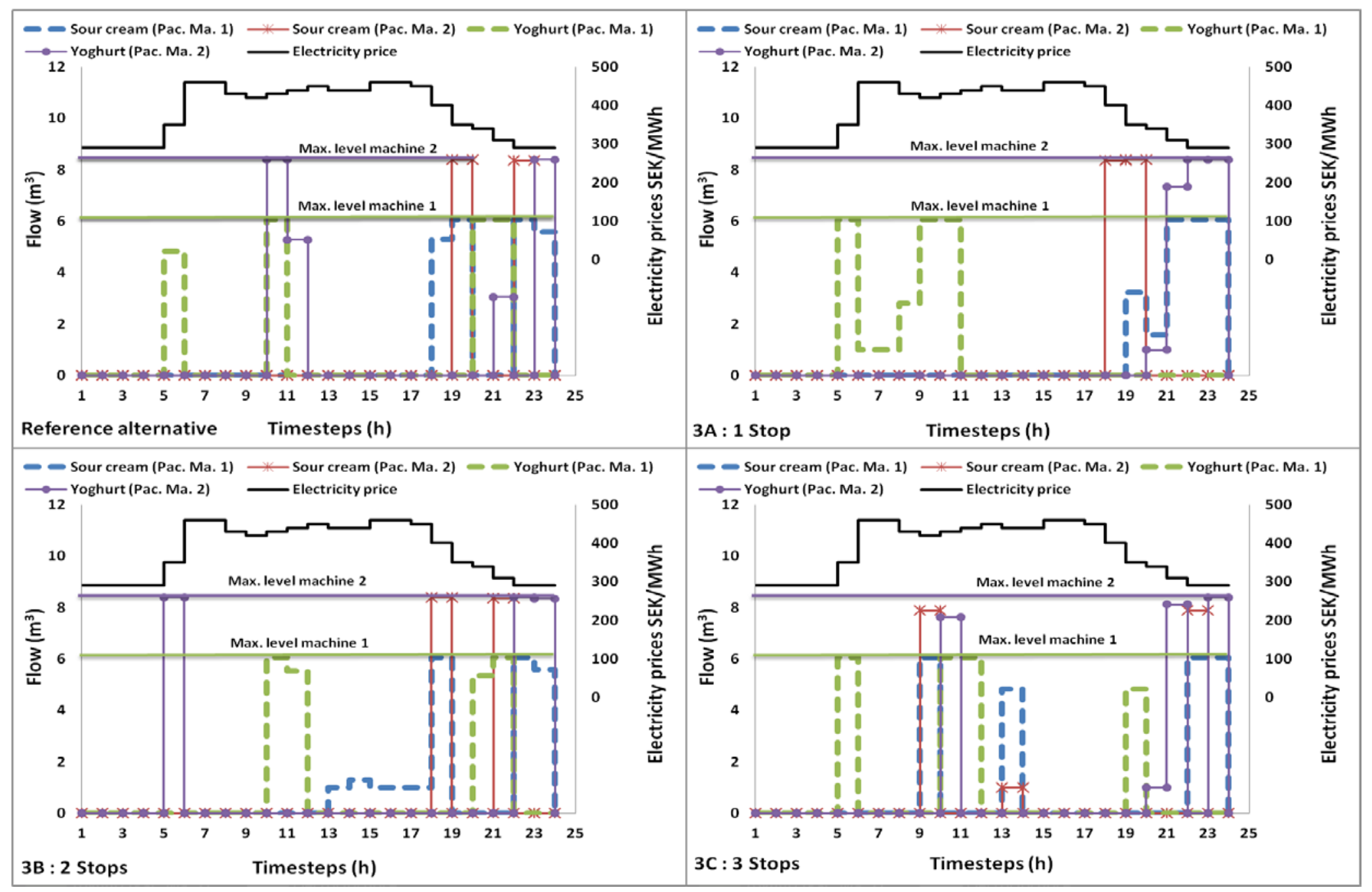

Figure 5. Results from the optimisation for the Reference alternative and Alternative 3, which represent the limitation of shutdowns, where Alternatives 3A, 3B and 3C represent one shutdown, two shutdowns and three shutdowns respectively. 


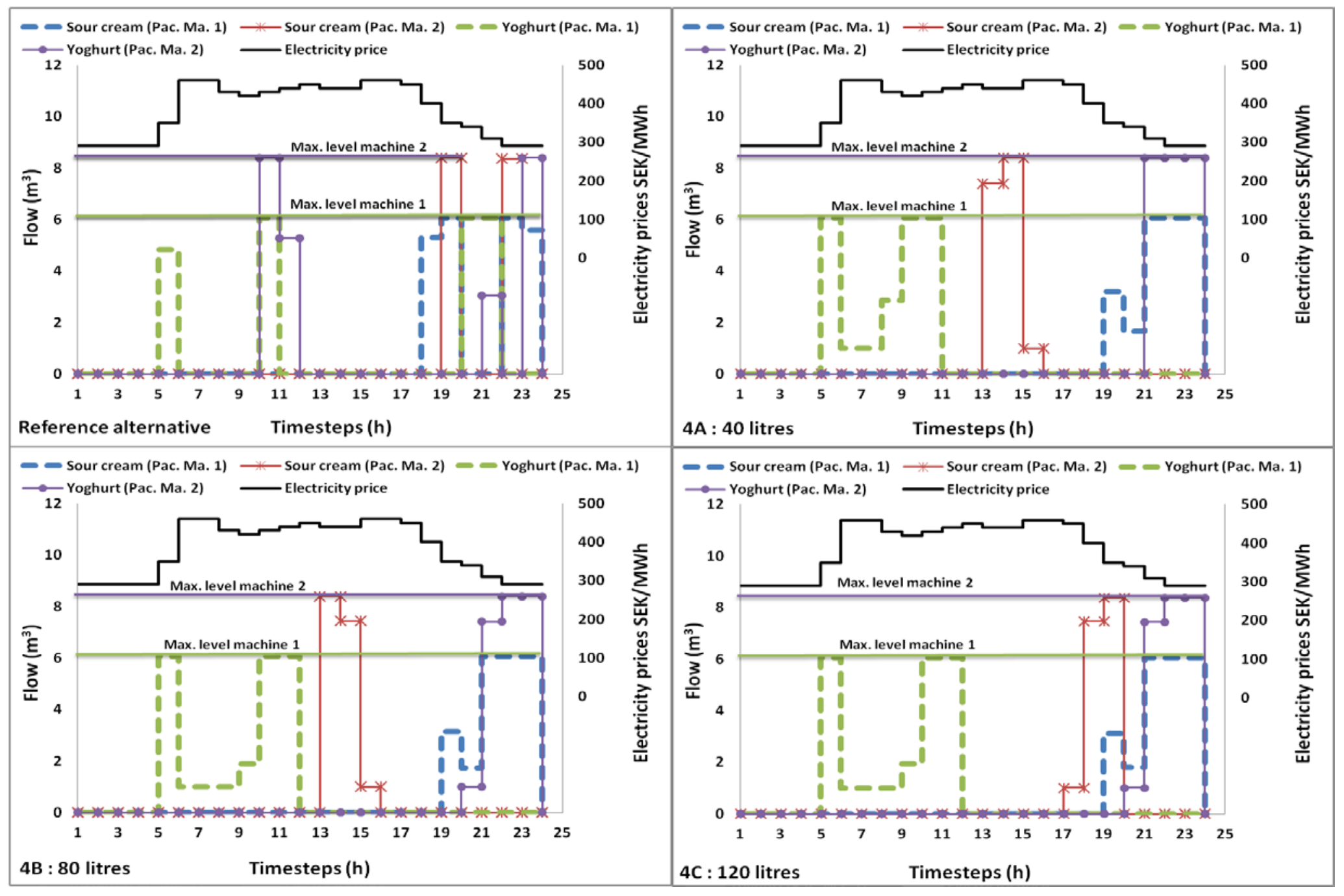

Figure 6. Results from the optimisation for the Reference alternative and Alternative 4, which represent a shutdown that is associated with a waste flow, where Alternatives 4A, 4B and 4C represent a waste of 40, 80 and 120 litres of yoghurt or sour cream respectively for each shutdown. 
Table 1. The input data for the different processes in the model [4].

\begin{tabular}{|c|c|c|c|c|c|}
\hline & $\begin{array}{l}\text { Electricity } \\
\left(\mathrm{kWh} / \mathrm{m}^{3}\right)\end{array}$ & $\begin{array}{c}\text { Steam } \\
\left(\mathrm{kWh} / \mathrm{m}^{3}\right)\end{array}$ & $\begin{array}{l}\text { Ice water } \\
\left(\mathrm{kWh} / \mathrm{m}^{3}\right)\end{array}$ & $\begin{array}{c}\text { Max } \\
\text { production } \\
\left(\mathrm{m}^{3}\right)\end{array}$ & $\begin{array}{l}\text { Incubation time/ } \\
\text { max storage time } \\
\text { (h) }\end{array}$ \\
\hline Silo tanks & - & - & 4.66 & - & 20 \\
\hline Separator ${ }^{a}$ & 1.049 & - & - & - & - \\
\hline Homogenizer & 9.2 & - & - & - & - \\
\hline Pasteurizer 1 & - & 5.2 & 7.0 & 40 & - \\
\hline Cream pasteurizer & - & 14.0 & 70.0 & 7.5 & - \\
\hline Cream tanks & - & - & - & - & 15 \\
\hline Pasteurizer 2 & - & 38.0 & 14.0 & 20.0 & - \\
\hline Silo (Cream milk) & - & - & - & - & 15 \\
\hline Silo (Whole milk) & - & - & - & - & 15 \\
\hline Silo (Skim milk) & - & - & - & - & 15 \\
\hline Incubation (Yoghurt) ${ }^{\mathrm{b}}$ & - & - & - & - & 5 \\
\hline Incubation (Sour cream) ${ }^{\mathrm{b}}$ & - & - & - & - & 8 \\
\hline Pasteurizer and homogenizer & 9.2 & 38.0 & 7.0 & 20.0 & - \\
\hline Cooling (Yoghurt) & - & - & 25.7 & - & - \\
\hline Cooling (Sour cream) & - & - & 25.7 & - & - \\
\hline Buffer (Yoghurt) & - & - & - & - & 6 \\
\hline Buffer (Sour cream) & & & & & 15 \\
\hline Packaging machine 1 & 5.5 & - & - & 6.06 & - \\
\hline Packaging machine 2 & 5.5 & - & - & 8.4 & - \\
\hline
\end{tabular}

${ }^{a} 10.39 \%$ of the whole milk is separated as cream and the rest is skim milk.

${ }^{\mathrm{b}}$ In the model there is no production of yoghurt in the packaging machines during the first 5 hours and no production of sour cream in the packaging machines during the first 8 hours. This is due to the fact that the incubation times for these products are 5 and 8 hours, respectively. 
Table 2. The analysed alternatives.

\begin{tabular}{|l|l|l|l|l|}
\hline Alternative Change & A & B & C & Units \\
\hline $\mathbf{1}$ & 50 & 60 & 70 & $\%$ \\
\hline $\mathbf{2}$ & 200 & 400 & 600 & SEK \\
\hline $\mathbf{3}$ & 1 & 2 & 3 & Stop(s) \\
\hline $\mathbf{4}$ & 40 & 80 & 120 & Litres \\
\hline
\end{tabular}

Table 3. System costs and peak demand at the dairy for Alternative 1.

\begin{tabular}{|l|l|l|l|l|l|l|}
\hline \multirow{2}{*}{$\begin{array}{c}\text { Analysed } \\
\text { Alternatives }\end{array}$} & \multicolumn{2}{|l|}{ System costs } & \multicolumn{2}{l|}{$\begin{array}{l}\text { Peak (electricity } \\
\text { demand) }\end{array}$} & \multicolumn{2}{l|}{ Peak (steam demand) } \\
\cline { 2 - 8 } & SEK & $\begin{array}{l}\text { Percentage } \\
\text { increase or } \\
\text { decrease }\end{array}$ & $\mathbf{k W}$ & $\begin{array}{l}\text { Percentage } \\
\text { increase or } \\
\text { decrease }\end{array}$ & $\mathbf{k W}$ & $\begin{array}{l}\text { Percentage } \\
\text { increase or } \\
\text { decrease }\end{array}$ \\
\hline Reference & 428,001 & 0 & 989 & 0 & 1,033 & 0 \\
\hline $\mathbf{1 A}$ & 428,045 & 0.0103 & 1,166 & 18 & 1,640 & 59 \\
\hline $\mathbf{1 B}$ & 428,054 & 0.0124 & 1,166 & 18 & 1,640 & 59 \\
\hline $\mathbf{1 C}$ & 428,062 & 0.0143 & 1,106 & 12 & 1,617 & 57 \\
\hline
\end{tabular}


Table 4. System costs and peak demand at the dairy for Alternative 2.

\begin{tabular}{|l|l|l|l|l|l|l|}
\hline \multirow{2}{*}{$\begin{array}{c}\text { Analysed } \\
\text { alternatives }\end{array}$} & System costs & \multicolumn{2}{l|}{ Peak (electricity demand) } & Peak (steam demand) \\
\cline { 2 - 7 } & SEK & $\begin{array}{l}\text { Percentage } \\
\text { increase or } \\
\text { decrease }\end{array}$ & $\mathbf{k W}$ & $\begin{array}{l}\text { Percentage } \\
\text { increase or } \\
\text { decrease }\end{array}$ & $\mathbf{k W}$ & $\begin{array}{l}\text { Percentage } \\
\text { increase or } \\
\text { decrease }\end{array}$ \\
\hline Reference & 428,001 & 0 & 989 & 0 & 1,033 & 0 \\
\hline 2A & 428,819 & 0.19 & 918 & -7.2 & 1,010 & -2.2 \\
\hline 2B & 429,619 & 0.38 & 878 & -11.2 & 1,520 & 47.2 \\
\hline 2C & 430,427 & 0.57 & 878 & -11.2 & 1,010 & -2.2 \\
\hline
\end{tabular}

Table 5. System costs and peak demand at the dairy for Alternative 3.

\begin{tabular}{|c|c|c|c|c|c|c|}
\hline \multirow[b]{2}{*}{$\begin{array}{c}\text { Analysed } \\
\text { alternatives }\end{array}$} & \multicolumn{2}{|c|}{ System costs } & \multicolumn{2}{|c|}{ Peak (electricity demand) } & \multicolumn{2}{|c|}{ Peak (steam demand) } \\
\hline & SEK & $\begin{array}{l}\text { Percentage } \\
\text { increase or } \\
\text { decrease }\end{array}$ & $\mathbf{k W}$ & $\begin{array}{l}\text { Percentage } \\
\text { increase or } \\
\text { decrease } \\
\end{array}$ & $\mathbf{k W}$ & $\begin{array}{l}\text { Percentage } \\
\text { increase or } \\
\text { decrease }\end{array}$ \\
\hline Reference & 428,001 & 0 & 989 & 0 & 1,033 & 0 \\
\hline 3A & 428,022 & 0.004 & 853 & -14 & 1,520 & 47 \\
\hline 3B & 428,009 & 0.002 & 929 & -6 & 1,010 & -2 \\
\hline $3 C$ & 428,004 & 0.001 & 1,164 & 18 & 1,639 & 59 \\
\hline
\end{tabular}


Table 6. System costs and peak demand at the dairy for Alternative 4.

\begin{tabular}{|l|l|l|l|l|l|l|}
\hline \multirow{2}{*}{$\begin{array}{c}\text { Analysed } \\
\text { alternatives }\end{array}$} & \multicolumn{2}{|l|}{ System costs } & \multicolumn{2}{l|}{ Peak (electricity demand) } & \multicolumn{2}{l|}{ Peak (steam demand) } \\
\cline { 2 - 7 } & SEK & $\begin{array}{l}\text { Percentage } \\
\text { increase or } \\
\text { decrease }\end{array}$ & $\mathbf{k W}$ & $\begin{array}{l}\text { Percentage } \\
\text { increase or } \\
\text { decrease }\end{array}$ & $\mathbf{k W}$ & $\begin{array}{l}\text { Percentage } \\
\text { increase or } \\
\text { decrease }\end{array}$ \\
\hline Reference & 428,001 & 0 & 989 & 0 & 1,033 & 0 \\
\hline 4A & 428,861 & 0.2 & 989 & 0 & 1,033 & 0 \\
\hline 4B & 429,675 & 0.4 & 878 & -11.2 & 1,212 & 17 \\
\hline 4C & 430,495 & 0.6 & 878 & -11.2 & 1.010 & -2 \\
\hline
\end{tabular}

